ISBN 978-93-84422-79-0

9th International Conference on Recent Trends in Science Engineering, Computers and Technology

(RTSECT-2017)

Singapore Aug. 10-11, 2017

\title{
Development of Web-Based GIS Cloud Apllication for Natural Resources Information System in West Sumatera
}

\author{
Yuhendra $^{1}$ and Eva Yulianti ${ }^{2}$ \\ ${ }^{1,2}$ Department of Informatics, Faculty of Industrial Engineering, Padang Institute of Technology, Indonesia \\ E-mail:yuhendrasan@gmail.com / yuhendra@ieee.org
}

\begin{abstract}
The cloud geographical information system (GIS) has been an important development direction of GIS in recent years. To deploy GIS on cloud and enable users to enjoy the geographical information services at any time and any place is becoming prominent, as companies and institutions around the globe realize the significance of cloud GIS applications in Spatial Decision Support Systems. We use web-based Cloud GIS (also called internet GIS or on line GIS) application for standards software packages to develop an interactive web mapping portal for spatial analysis. The proposed of research address was development of web-based clolud GIS application and identifying potential areas for tourism natural resources in South Solok part in West Sumatera Province. It was such as culture, natural, and agro tourism. Since the South Solok has great potential in many object for domestic and international tourism destination, it is a need to develop system as as for resources information system tools in decision support system (DSS) to helping and managing the company's geothermal resources for key decision making, planning and also provides the users with an innovative and interactive way to access the spatial content over the internet/intranet. The system will be implemented by the architecture of cloud computing, cloud is used as a place to store the data, applications, and others to facilitate the exploration of natural resources tourism. The result application provides a variety of functionalities such as; browse map mode, querying, navigation system, user requiring service, search region and displaying tourism natural resources areas, map editing and printing from the web interface.
\end{abstract}

Keywords: south solok, natural resources, cloud GIS, decision support system

\section{Introduction}

South Solok regency, West Sumatera Province is one of the districts that has a lot of regional income that will be ready to increase and accelerate economic growth in tourism sector. Tourism is an inventory of the country that is still hidden and has the potential to be developed in the effort to explore the sources of local income, especially in the potential tourism of the district of Solok Selatan. It is come from natural, historical and cultural tourism in spreading in other areas. The constraints experienced during this time both in the community and government such as the lack of entertainment, incomplete information in the form of locations and routes, attractions that are not managed properly. Currently, in the e-goverment South Solok regency website there is no information about the mapping area data tourism destination. In order to make it easier mapping for people to get data and find the location of tourism, we have developes the geographic information systems (GIS). Since it is a current technology to handle various spatial maps and databases. Integration of GIS technology into cloud, cloud computing is associated with a new paradigm for the provision of computing. This paradigm shifts the location of infrastructure from desktop to the network to reduce the costs in management of hardware and software resources. Cloud computing is network based computing in which shared resources, software and information are provided to different clients on demand.

Over the recent years, advancement of internet has become the major carrier of information around the globe, offering increased accessibility and mobility. Beyond data sharing, the integration of the World-Wide Web 
(WWW) with Web-based GIS System technologies (Web GIS) is becoming prominent, as companies and institutions around the globe realize the significance of Web GIS applications in Spatial Decision Support Systems. A many researchers have addressed problems about studied the importance of cloud-computing in various sectors for storing large amount of data and GIS as a separate concept in viewing the spatial maps. And also an enormous increase in the number of web-based applications that use techniques derived from cloud GIS (Singh et al.,2012; Ebenzer, 2012, Kodge et al., 2012). In application, a GIS should be capable of six fundamental operations in order to be useful for finding solutions to real-world problems, such as capture data, store data, query data, analyze data, display data and output data.

A Web-Based cloud GIS (also called internet GIS or on line GIS) is a network-based geographic information service that utilizes both wired and wireless Internet to access geographic information and analytical tools in delivering GIS services (Kouyoumjian, 2011, Kodge, et.al, 2012 ). Tom Bernhardsen (1999) defines GIS as a system that captures, stores, manage, manipulates, retrieve and analyze geographic or spatial data through computer hardware/software and other cartographic special devices to create map products. This technology is capable to managing, analyzing, integrating and displaying various forms of geographically referenced information. The spatial information comes from digital mapping being used in spatial decision support systems $(D S S)$. The ability of GIS to integrate maps and data bases, using the geography as the common feature among them has been extremely effective in the context of planning development. And also Web-based GIS applications could provide more sophisticated cartography and spatial visualization features (Lu et al., 2003).

In this work, the design and architecture of the Web Map Server (WMS) is presented and discussed. WMS provides data sharing, visualization of geospatial data and spatial decision support services for environmental planning and management (Sakamoto \& Fukui, 2004). The main contribution of this work is that, by using Integration of GIS technology into cloud together emerges as a crucial model for capturing, uploading and maintaining the new promising web applications in natural resources application. The application of Information Technology in various filed has brought greatimpact especially in natural tourism sector. The proposed approach of natural resources information development and integration system ensures that complete tourism areas with related data on cloud database can be integrated into spatial maps through GIS technologies, to organize, accumulate, and administer geospatial data in a cloud database. The researchers explain about the cloud computing as advanced approach in managing the data, storing and from desktops to huge data repositories at low cost sites. This approach represents application programs and data as service through Internet. Development of GIS based cloud computing to enhance server's efficiency, reducing the computational cost and storage, rapid processing and computing of the data unlimitedly, huge amount of storage capacity and to increasing

the efficiency and consistency of the system. In this research, authors explain the advantages of using cloud computing technology to help in natual resources tourism development of Solok Selatan Regancy, West Sumatera.

\section{Study Area}

The study area in this case study is located Solok Selatan regency in the southern of West Sumatra Province. It is nerby is nearby to Bukit Kunyieng, Siruek and Bukit Gadang. Kabupaten Solok Selatan has about 144,000 residents and an elevation of 261 meters. A location with the geographic coordinates of Latitude $1^{\circ} 14^{\prime}$ $\left(1.2333^{\circ}\right)$ from south and Longitude $101^{\circ} 25^{\prime} 1.2^{\prime \prime}\left(101.417^{\circ}\right)$ east.

\section{Web Development}

A WMS is a standard protocol for serving geo-referenced map images over the Internet that was generated by a Map Server using GIS data. The Map Server is an Open Source platform environment for publishing spatial data and interactive mapping applications to the web have been used as spatial data server. It creates map images from spatial information stored in digital format. Map Server consists of three different components: map file, template files and the CGI program. The map file needs to set cartographic parameters, cartographic objects, data loading, classification, displaying and querying and graphic elements definition and use. It is implemented using Map Server software's built-in object oriented scripting language with which it is possible to design how to create and use the maps and their layers.

For users wishing to develop web-based mapping applications, a number of web GIS application development tools are available nowadays such as open source (Singh, 2012) and commercial platform have become more popular. 
Web-based GIS applications have client/server architecture, which has a client side and a server side. In this architecture users interact with the GIS application through a browser. The web browser is a client which performs presentation functions while the web server is to be considering as a remote server which performs data storage, extraction and processing (Alesheikh et al. 2002; Soltanieh et al. 2003). On the server side sides the GIS database and applications to process the user's request. On the client side is a user interface within a web browser. Whenever a user submits a request, the server processes the request with the GIS application program and returns the result to the user. Other Client side platform desktop GIS software program commercial software packages such as ESRI ArcView and MapInfo Professional are used. Since it is offer robust GIS capabilities and the programs enable users to view, edit geographic data, produce high quality cartographic maps, and perform advanced spatial analysis. It is make it possible to deliver GIS maps and data to individuals using only a web browser. Users may access these applications over the Internet or via a local Intranet connection. The maps may either be static graphics (such as .pdf or .jpg files), or interactive applications that allow users to zoom in and out, turn layers on and off and perform basic inquiries. Interactive web-based GIS applications utilize central map and web server technologies to manage information requests and deliver maps and data to remote users. With webbased GIS, the technology has evolved from web map publishing to web-GIS mapping.

In figure 1, a mapping application is using dynamic web browser. It is enable to choose features that will be displayed, such as the scale, location etc. And then the web browser displays the map as an image. By changing the parameters, users can generate and view a new map. The server side has geographic data, GIS soft wares, and an interface program. The flow processing of GIS design as shown in the figure 2. At the basic level, publishing geospatial data on the internet needs a web server and a set of maps. The maps should be supplied in a format web browser.

The standard web browser communicates with the web server is called Hyper-Text Transfer Protocol (HTTP). The HTTP is the standard for transferring World Wide Web documents. In a dynamic configuration side, the HTTP server is connected to a Map Server with GIS software installed, which is able to generate maps on request. This request is processed by the GIS server. The result will be sent to the browser. A server with GIS functions is called an Internet Map Server. It is a standard protocol for serving geo-referenced map images over the Internet. Since there are many ways to link the HTTP server with the GIS server through interface programs in this research.

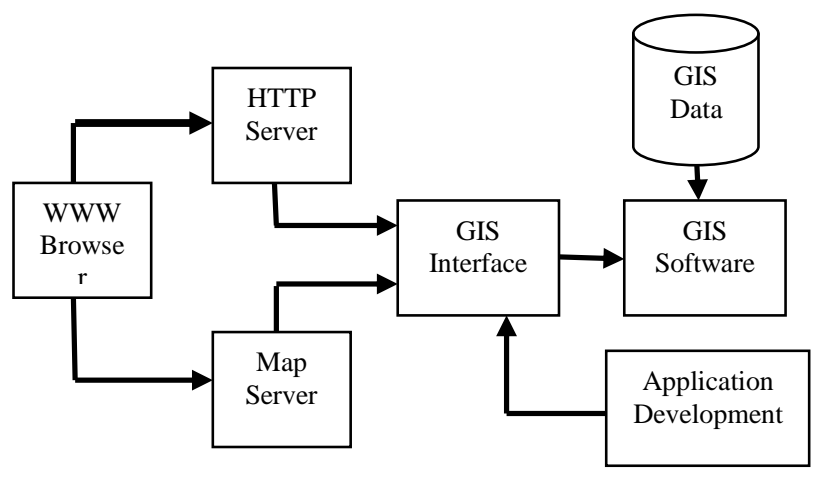

Fig. 1: Server side mode (Yuhendra, 2015)

\subsection{GIS Cloud Based}

Softare as a service (SaaS) based GIS cloud is defined as a software application delivery model (Figure 3), where a software vendor deploys and hosts software applications in a multi-tenant (cloud) platform for its customers to operate the application over the Internet as services. Software as a Service based GIS cloud allows anyone with a browser to instantly use desktop GIS. This innovative cloud computing technology gives subscribers full access to GIS tools such as data creation, analysis, editing and visualization (Liu et al., 2010). Comparing with conventional GIS software, SaaS based GIS cloud has some unique features. Instead of being installed on premise, SaaS applications are usually hosted at the service provider's network, delivered as web applications, and serve as services for multiple users. This on-demand and multi-user service delivery model is well suited for software cloud, as it does not require the deployment of a large infrastructure at the client's location. On the other hand, SaaS applications can be deployed in a cloud computing environment and accessed through Internet by web browsers. It eliminates or drastically reduces the upfront commitment of resources. SaaS 
applications can be deployed with minimal effort and be available in a very short time to a large group of users, SaaS model due to its varied advantage, is quite attractive to enterprises. Figure 1 shows multi-user SaaS model (Pervez et al., 2010).

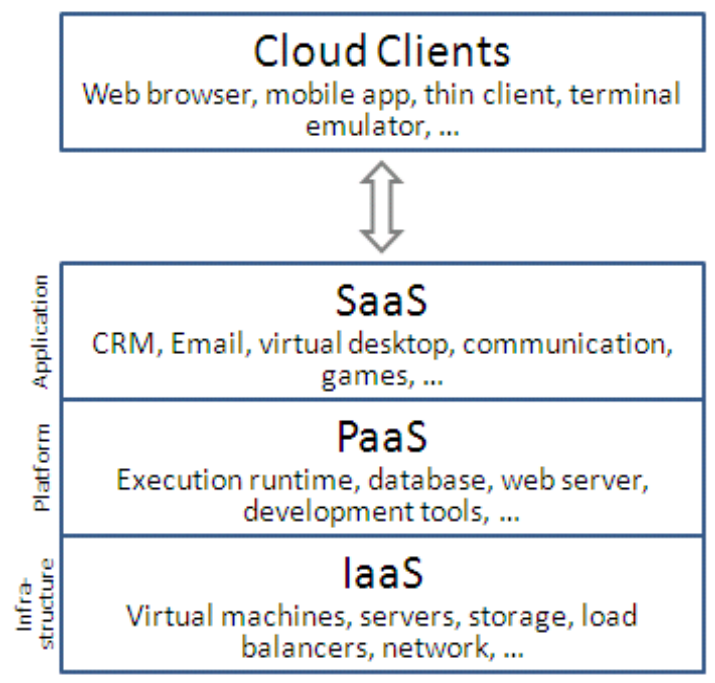

Fig. 2:. Cloud computing Model Service (Kouyoumjian, 2011)

Comparing with conventional GIS software, SaaS based GIS cloud has some unique features. Instead of being installed on premise, SaaS applications are usually hosted at the service provider's network, delivered as web applications, and serve as services for multiple users. This on-demand and multi-user service delivery model is well suited for software cloud, as it does not require the deployment of a large infrastructure at the client's location. On the other hand, SaaS applications can be deployed in a cloud computing environment and accessed through Internet by web browsers. It eliminates or drastically reduces the upfront commitment of resources. SaaS applications can be deployed with minimal effort and be available in a very short time to a large group of users, SaaS model due to its varied advantage, is quite attractive to enterprises.

Application Layer as a service (Paas) is embedded with an operating system platform for hosting various applications. And Physical Layer as a service (Iaas) which represents the infrastructure involved in complete development of the web application is built virtually by presenting the virtual servers as a service to users.

\section{Web and Map Application Development}

Development standards describe the choice of an appropriate development technology for the web application. Web applications developed in PHP (Hypertext Preprocessor) and HTML (Hypertext markup Language), which is an open source technology used to develop web applications. A web application often stores temporary data in files or communicates with other web applications over the web. For Common communication protocols used to enhance the performance of the web application is HTTP (Hypertext Transfer Protocol). Use of a common communications protocol enhances the performance of the web application because the information contained in the application need not be converted into the application data format. Data sent by the other web application or service can be used directly by the caller web application and this enhances the response time to users. The content of the internet map that would be seen by a user was developed using Map server application. The Map Server is an Open Source platform environment for publishing spatial data and interactive mapping applications to the web have been used as spatial data server. It creates map images from spatial information stored in digital format. All information concerning the map to be served, its color properties, the layers definition and many other attributes are configured from the Map file.

Map Server consists of three different components: map file, template files and the CGI program. The map file needs to set cartographic parameters, cartographic objects, data loading, classification, displaying and querying and graphic elements definition and use. It is implemented using Map Server software's built-in object oriented scripting language with which it is possible to design how to create and use the maps and their layers. In the operation, Map Server can operate in two different modes: Map Script APIs and via a CGI executable. In this mode, the rendering depends on two files: the configuration file "map file" and the HTML template. The map file 
is an ASCII file which defines the data objects used to produce the map. The template file is an HTML file with tagged replaceable parameters enclosed within square brackets. Map Server processes each replaceable parameter in the template file and returns the result. In design of data format from raster map to digital map is created in MapInfo Professional 10.5 (figure 4). A digitations map is used in to register digital images through the software applications. This step was very important work to input the position control point in geographic coordinates. To creates the map areas using the layers tool that is available facilities. We can add a new map layer and the combines with other layer with the click on menu that created region area map. In digitations process, we must convert the image file to shape file format using a Map Server through the Map Info software.

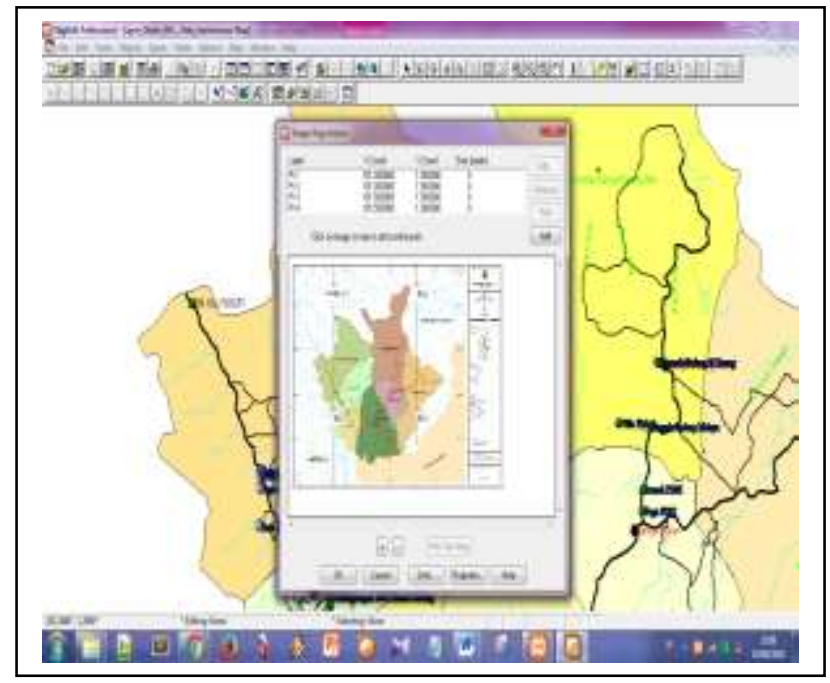

Fig. 3: Digitation map Process

\section{Result}

The Web-based GIS mapping of natural resources information system was developed in Figures (5-7).

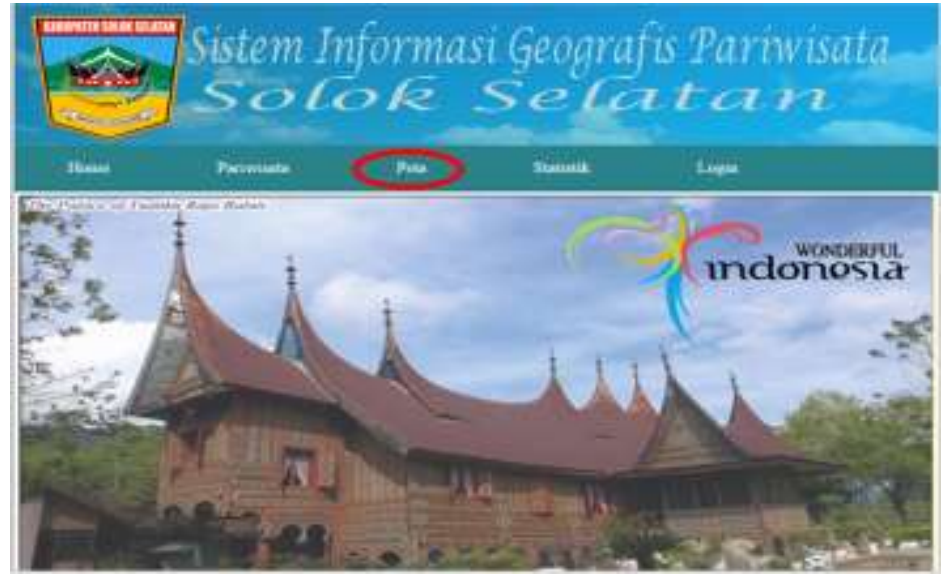

Fig. 4: Web-based GIS mapping portal

It is application is to provide a powerful application with a simple and user friendly interface which a user with no GIS experienc e and knowledge of using the GIS tools should be able to navigate through the system without any confusion. The portals service (figure 5) provides a variety of information such as; list of region, gallery, map and contact. A user can browser a list of regions area contain the information tourism destination. The application is easy to use and it provides a valuable resource for accessing spatial datasets. In order to be useful for finding solutions to real-world problems. A GIS should be able to capture data, store, query, analyze, display and output data. 


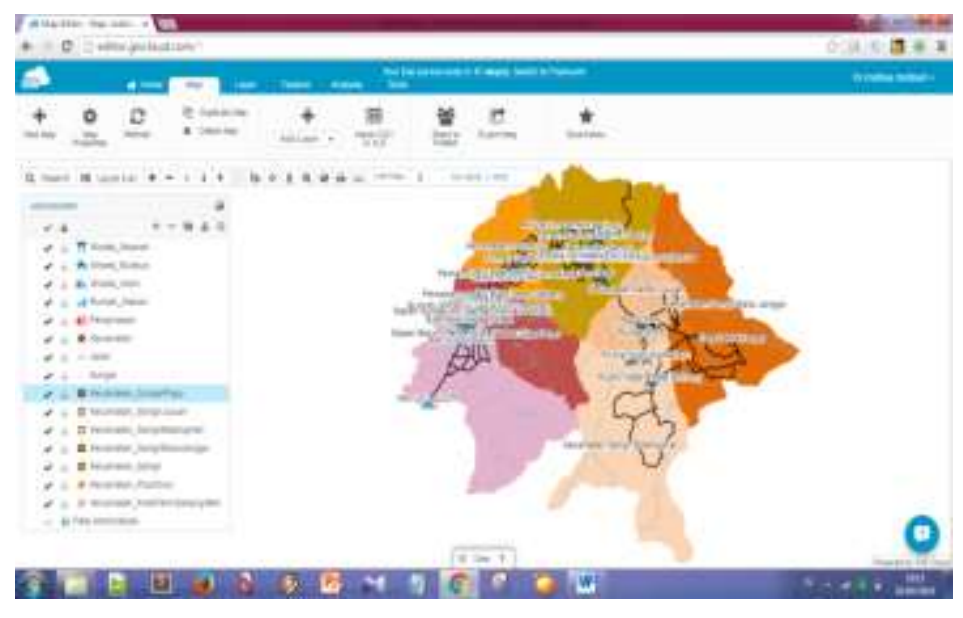

Fig.5: Web-based GIS Map Display

In map display (figure 6), a variety of tool functionalities are divided into the following categories as follows;

- Map Navigation Tools (Mode Browse) - Zoom In, Zoom Out, Zoom to Full Map, Re-center

- Attribute Data Retrieval Tools - Identify, Select layer, Query, information layer

- Other Tools -Scale, Select Feature, and Print

A map display give information about the natural resources tourism in each area in South Solok regancy such historical, cultural, and natural tourism destination.
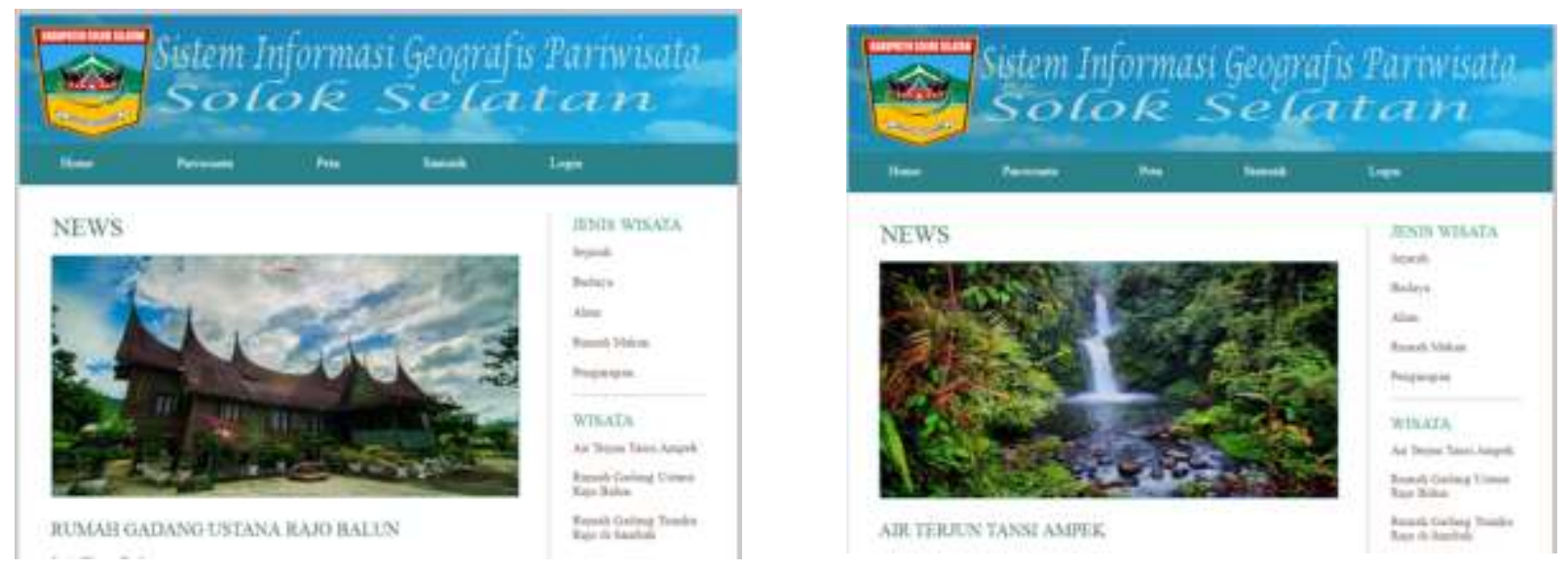

Fig.6. Natural Resources Tourism Information

In the left side map, information about layer is given. It is consist of a layers south Solok regancy area ( Ko o gadang, sungai pagu, pauh duo, sangir jujuan, sangir balai janggo, sangir, sangir batang hari). On right side, areas location based color region. A user can easily to find the distribution mapping of natural resources information each location. We are only selected a layer menu or write the name of area in button search facility. And others facilities, we can retrieve information using identify.

\section{Conclusion And Future Research}

This paper presented WMS, a web-based GIS cloud for standards software tools. Careful integration of these packages and the use of state-of-the-art web programming techniques resulted in a powerful and robust Web GIS. This system was developed to make identification and mapping distribution of natural resources tourism destination. A User can also easily find information on target areas using the Internet and save the results as a 
figure or as spreadsheet data. During the research project a geothermal resource database was created. The database in conjuction with the application allows various users to access information quickly and efficiently. In the future, natural resources information system is being improved for advanced analysis must be combine with remote sensing application using data satellite will be investigated.

\section{Acknowledgements}

The research is supported by Indonesia Higher of Education (DIKTI). And especially for anonymous reviewer for valuable comments and suggestions..

\section{References}

[1] Ebenzer, Y.O, 2012. Developing resources database with Nigeriasat-1 Satellite data and geographical information system, The Egyptian journal of remote sensing and space sciences ,15, 207-214.

[2] Puyam S. Singh, et.al., 2012. Development of a Web Based GIS Application for Spatial Natural Resources Information System Using Effective Open Source Software and Standards, Journal of Geographic Information System, 2012, 4, 261-266.

[3] Martin,D, Atkinson, P, 2000. Editorial: Innovation in GIS application, Journal of Computers, Environments and Urban Systems, Vol. 24,Issue 2, pp. 61-64.

[4] Peng, Z. R. and Tsou, M.2003 Internet GIS: Distributed eographic Information Services for the Internet and Wireless Networks John Wiles and Sons Inc. Hoboken, NJ, USA. p. xxv.

[5] Ramachandra T. V. 2007 Solar energy potential assessment using GIS .Energy Education Science and Technology 18(2): 101-114.

[6] Tsou, M. (2004). Present Realities and the Future of Internet GIS.GISDevelopment

[7] Kodge B. G, and Hiremath P. S. 2012.Web based Geo-Spatial and Village Level Information Extraction System using FOSS, Journal of Advances in Information Technology, 3(4),222-227

[8] Caldeweyher, D., Jinglan Zhang \& Pham, B., 2006. OpenCIS-open source GIS-based Web community information system. International Journal of Geographical Information Science, 20(8), 885-98.

[9] Soltanieh, S.M.K., Alesheikh, A.A. \& Mohammadi, H., 2003. Design and implementation of a web-based GIS for the Iranian Roads Information. Available at: http://www.gisdevelopment.net/technology/gis/mi0363.htm

[10] Alesheikh, A., Helali, H. \& Behroz, H., 2002. Web GIS: Technologies and Its Applications. Symposium on Geospatial Theory, Processing and Applications, 15.

[11] Chan Kim, H., Baek, S.G.,Park, J., 2010. Construction of Geothermal Information Database for Korea Based on Web GIS. Proceedings World Geothermal Congress 2010 Bali, Indonesia.

[12] Lu, C. T., Kou, Y.,Wang, H., Shekhar, S., Zhang, P. \& Liu, R., “Two web-based spatial data visualization and mining systems: Mapcube \& Mapview”, 2003, Proc. Next-Generation Geospatial Information Systems (NG2I’03).

[13] Sakamoto, A. \& Fukui, H., "Development and application of a livable environment evaluation support system using Web GIS”, Journal. of Geographical Systems, 6(2), 2004, pp. 175-195. 